\title{
Nanostructure-Assisted Phonon Scattering in Lead-Free Thermoelectric Materials: A TEM Investigation of the SnTe System
}

\author{
Fengyuan Shi ${ }^{1}$, Shih-Han Lo ${ }^{1}$, Gangjian Tan ${ }^{2}$, Li-Dong Zhao ${ }^{2}$, Mercouri G. Kanatzidis ${ }^{2,3}$ and Vinayak \\ Dravid $^{1}$
}

1. Department of Materials Science and Engineering, Northwestern University, Evanston, Illinois 60208, United States

2. Department of Chemistry, Northwestern University, Evanston, Illinois 60208, United States

3. Materials Science Division, Argonne National Laboratory, Argonne, Illinois 60439, United States

We have used HRTEM and STEM EDX to determine the important roles of endotaxial nanostructures in a lead-free thermoelectric (TE) material, $\mathrm{SnCd}_{0.03} \mathrm{Te}$, with $2 \% \mathrm{CdS}$ and $2 \% \mathrm{ZnS}$; where maximum $Z T s$ of $\sim 1.3$ and $\sim 1.1$ are achieved at $873 \mathrm{~K}$, respectively. TE materials convert wasted heat into electric energy. The conversion efficiency of TE materials is characterized by a dimensionless figure of merit $Z T$ $=S^{2} \sigma T / \kappa$, where $S$ is the Seeback coefficient, $\sigma$ is the electrical conductivity, $\mathrm{T}$ is the absolute temperature and $\kappa=\kappa_{e}+\kappa_{l}$ is the total thermal conductivity, including both electronic $\left(\kappa_{e}\right)$ and lattice $\left(\kappa_{l}\right)$ contributions. The record-high $Z T$ values resulting from nanoscale precipitates in p-type SnTe make SnTe-based materials ideal candidate for p-type lead chalcogenides [1-3] for high temperature thermoelectric power generation.

Figure 1 (a) and (b) show TEM images of nanostructured $\mathrm{SnCd}_{003} \mathrm{Te}$ with $2 \% \mathrm{CdS}$ and $2 \% \mathrm{ZnS}$, respectively. High-density nanoscale precipitates are found in both samples, presented by dark contrast in the images. These precipitates have two orthogonal symmetry variants, as schematically depicted in the inset in the upper right corner of Figure 1 (a). The SAD pattern along [001] orientation with an aperture that captures both the matrix and the precipitates shows only one set of Bragg reflection spots, indicating no notable difference in lattice spacing between the matrix and the precipitate because of their small lattice mismatch. We have also employed statistical analysis to obtain the size distribution of $\mathrm{SnCd}_{0.03} \mathrm{Te}-2 \% \mathrm{CdS}$ and $\mathrm{SnCd}_{0.03} \mathrm{Te}-2 \% \mathrm{ZnS}$ samples along [001] orientation based on Figure 1(a) and (b), respectively. In Figure 1 (c), the histograms of these two samples show notable difference. We found that for $\mathrm{SnCd}_{0.03} \mathrm{Te}-2 \% \mathrm{CdS}$, most nanoscale precipitates range from 3 to $4 \mathrm{~nm}$ in size, but for $\mathrm{SnCd}_{0.03} \mathrm{Te}-2 \% \mathrm{ZnS}$, the size of the majority precipitate falls within 4.5 to $7 \mathrm{~nm}$ range. Comparing $\mathrm{ZnS}$ and $\mathrm{CdS}$ cases, the larger size of $\mathrm{ZnS}$ nanostructures reduces interface area to the matrix, which in relative terms may result in reduced phonon scattering.

Figure 2 shows the HRTEM images and STEM EDX results of $\mathrm{SnCd}_{0.03}$ Te with $2 \% \mathrm{CdS}$ and $2 \% \mathrm{ZnS}$, respectively. An HRTEM image in Figure 2 (a) depicts a coherent nanoscale CdS precipitate embedded in the $\mathrm{SnCd}_{0.03}$ Te matrix along [001] orientation. No line defects or discontinuities are observed at the matrix/precipitate interface, confirming coherently strained nanoscale endotaxial precipitates. The STEM-EDS results in Figure 2 (b) indicate that the nanoscale precipitates are rich in $\mathrm{Cd}$ and S, but the matrix is devoid of sulfur. Similar analysis were applied to $\mathrm{SnCd}_{0.03}$ Te with $2 \% \mathrm{ZnS}$. Zn and S signals present only in the nanoscale precipitates, but not in the matrix.

High $Z T$ values of SnTe system with $\mathrm{CdS}$ and $\mathrm{ZnS}$ nanoscale precipitates are attributed to the enhanced phonon scattering at the nanoscale precipitates/matrix interfaces, which decreases the lattice conductivity $\kappa_{l}$ without degrading $\sigma$. The $Z T=1.3$ in $\mathrm{SnCd}_{0.03} \mathrm{Te}-2 \% \mathrm{CdS}$ is the record-high value for 
the p-type SnTe system. SnTe therefore as an attractive lead-free chalcogenide system is promising for high temperature power generating thermoelectric material. [4]

\section{References:}

[1] K. Biswas, J. He, I. D. Blum, C.-I. Wu, T. P. Hogan, D. N. Seidman et al., Nature, 489, (2012) 414-418.

[2] L. D. Zhao, H. J. Wu, S. Q. Hao, C. I. Wu, X. Y. Zhou, K. Biswas, et al., Energy \& Environmental Science, 6, (2013), 3346-3355.

[3] Y. Lee, S.-H. Lo, J. Androulakis, C.-I. Wu, L.-D. Zhao, D.-Y. Chung, et al., Journal of the American Chemical Society, 135, (2013), 5152-5160,.

[4] This work was supported as part of the Revolutionary Materials for Solid State Energy Conversion, an Energy Frontier Research Center funded by the U.S. Department of Energy, Office of Science, and Office of Basic Energy Sciences under Award Number DE-SC0001054. Transmission electron microscopy work was partially performed in the EPIC facility of the NUANCE Center at Northwestern University.
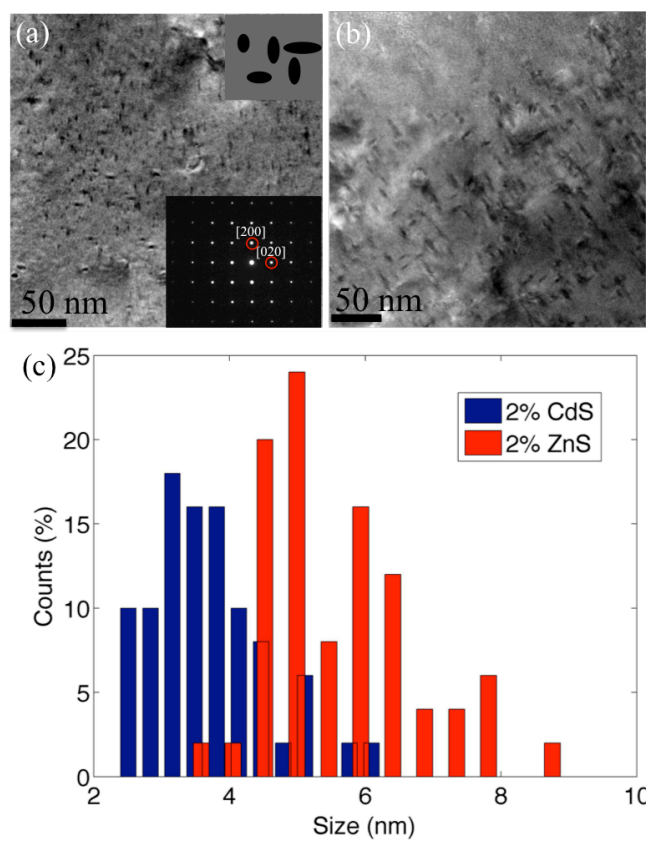

Figure 1 (a) and (b) are low magnification TEM images of $\mathrm{SnCd}_{0.03} \mathrm{Te}-2 \% \mathrm{CdS}$ and $\mathrm{SnCd}_{0.03} \mathrm{Te}-2 \% \mathrm{ZnS}$, respectively. The inset of the upper right corner of (a) is a schematic show of nano precipitates (black) embedding in the matrix (gray). The inset of the lower right corner of (a) is the SAD pattern taken along [001] direction. (c) is the histograms of precipitate size for $\mathrm{SnCd}_{0.03} \mathrm{Te}-2 \% \mathrm{CdS}$ (blue) and $\mathrm{SnCd}_{0.03} \mathrm{Te}-2 \% \mathrm{ZnS}$ (red), respectively.
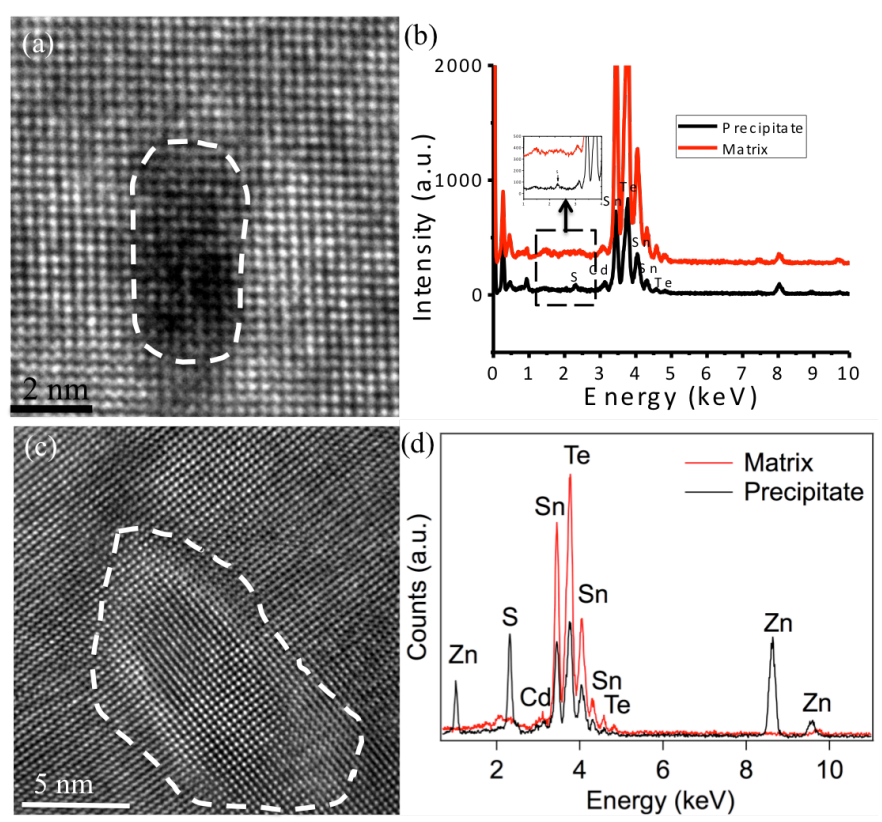

Figure 1 (a) HRTEM image of one nanoscale precipitate in $\mathrm{SnCd}_{0.03} \mathrm{Te}-2 \% \mathrm{CdS}$, highlighted by the dashed white line. (b) is STEM EDS of the $\mathrm{SnCd}_{0.03}$ Te- $2 \% \mathrm{CdS}$ sample. The spectra were taken at the precipitate and the matrix respectively. The Sn, $\mathrm{Te}, \mathrm{Cd}$, and $\mathrm{S}$ peaks were detected at the precipitate as shown in the inset. The matrix is with Sn, Te and Cd. (c) HRTEM image of one nano precipitate in $\mathrm{SnCd}_{0.03} \mathrm{Te}-2 \% \mathrm{ZnS}$ highlighted by the dashed white line. (d) is STEM EDS of the $\mathrm{SnCd}_{0.03}$ Te- $2 \% \mathrm{ZnS}$ sample. The spectra were taken at the precipitate and the matrix respectively. The precipitate has $\mathrm{Zn}$ and $\mathrm{S}$ signal, but the matrix has none. 